\title{
Nano-size uni-lamellar lipodisq improved in situ auto-phosphorylation analysis of $E$. coli tyrosine kinase using resonance
}

\section{Dear Editor,}

Among many of biophysical methods, ${ }^{19} \mathrm{~F}$ NMR spectroscopy has emerged as a powerful tool for characterizing protein structure, dynamics and function properties due to high intrinsic sensitivities of fluorine, $100 \%$ natural abundance of the NMR-active spin, the absence of any natural background in cells, and exquisite sensitivities of ${ }^{19} \mathrm{~F}$ chemical shift to environment (Shi et al., 2011; Shi et al., 2010). With evolved aminoacyl-tRNA synthase specifically recognizing the ${ }^{19} \mathrm{~F}$ containing unnatural amino acids, and

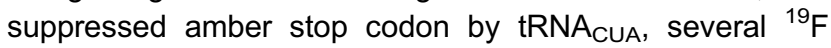
containing unnatural amino acids were incorporated into proteins in bacteria (Hammill et al., 2007; Jackson et al., 2007). Achievements of the protein-specific and site-specific ${ }^{19} \mathrm{~F}$ incorporation provided great opportunities for in cell or in situ protein structure, dynamic and function studies. Recently, ${ }^{19} \mathrm{~F}$-tfmF (trifluoromethyl-phenyl alanine) was incorporated to diacyl-glycerol kinase (DAGK) and we have achieved DAGK's conformation and dynamics analysis in native lipid bilayer environment using ${ }^{19} \mathrm{~F}$ solid state NMR directly through bacteria membrane separation, without laborious protein purification and lacking negative interferences from detergents (Shi et al., 2012). However, the lipid system separated from bacteria membrane spontaneously formed multi-lamellar vesicles (MLV) and high proportion numbers of membrane proteins located in inner vesicles, which provided physical barrier for accessibilities of soluble ligands or interaction partner proteins. The poly-styrenemaleic-acid (SMA) can wrap around lipid to form nanoparticles (with average size of $10 \mathrm{~nm}$ ), leaving the lipid bilayer a disc shape (lipodisq) (Knowles et al., 2009). The monodispersed lipodisq was reported to preserve the integrity of transmembrane proteins and form biocompatible, thermostable and soluble nano-particles (Orwick-Rydmark et al. 2012; Orwick et al., 2012). Very importantly, the two sides of the lipodisq can be accessed by soluble compounds or partner proteins, which provided great potential and convenience for biophysical analysis of membrane proteins in a minimized lipid environment.

Tyrosine phosphorylation is a reversibly post-translational modification that regulates many aspects of cellular functions (Hunter, 2009; Johnson and White, 2012). Tyrosine phosphorylation can be activated in both auto- and crosscatalytic by kinases in the presence of ATP and $\mathrm{Mg}^{2+}$, while the phosphorylation can be removed by phosphatases (protein tyrosine phosphatase, PTP). E. coli tyrosine kinase (ETK) is a transmembrane protein containing two transmembrane helices and a soluble kinase catalytic domain (ETK-CD). The ETK-CD had the auto-phosphorylation site Tyr574 and a tyrosine rich C-terminal tail (Lee et al., 2008) (Fig. 1A). Structure and function studies illustrated that the phosphorylated Tyr-574 consequently enabled cross-phosphorylation of the C-terminal tyrosine rich tail of ETK or tyrosine residues in other substrate proteins (Lee et al., 2008).

Previously, we have developed genetically encoded unnatural amino acid (3,5-difluorotyrosine, $\left.F_{2} Y\right)$ to implement site specific incorporation in ETK-CD (Li et al., 2013). Since the ${ }^{19} \mathrm{~F}$ spin was located neighboring to the active hydroxyl group, phosphorylation of the tyrosine immediately resulted in ${ }^{19} \mathrm{~F}$ chemical shift differences (i.e. $-134.54 \mathrm{ppm}$ for dephosporylated tyrosine and $-122.30 \mathrm{ppm}$ for phosphorylated tyrosine) (Li et al., 2013). However, the phosphorylation analysis of ETK-CD was implemented in vitro using purified proteins, while a crucial difference between in vitro and in situ conditions was expected. Especially, the in vitro sample contained rather high concentrations of macromolecules and lacked of native membrane environment (Barnes and Pielak, 2011). Here, phosphorylation property of Tyr574 in purified ETK-CD (Li et al., 2013) and full-length ETK (ETK-FL) in native $E$. coli membrane will be illuminated.

In this study, the $\mathrm{F}_{2} \mathrm{Y}$ was incorporated at Tyr574 site of ETK-FL protein through conventional unnatural amino acid incorporation methods. The ETK-FL protein with $\mathrm{His}_{6}$-tag in 
A

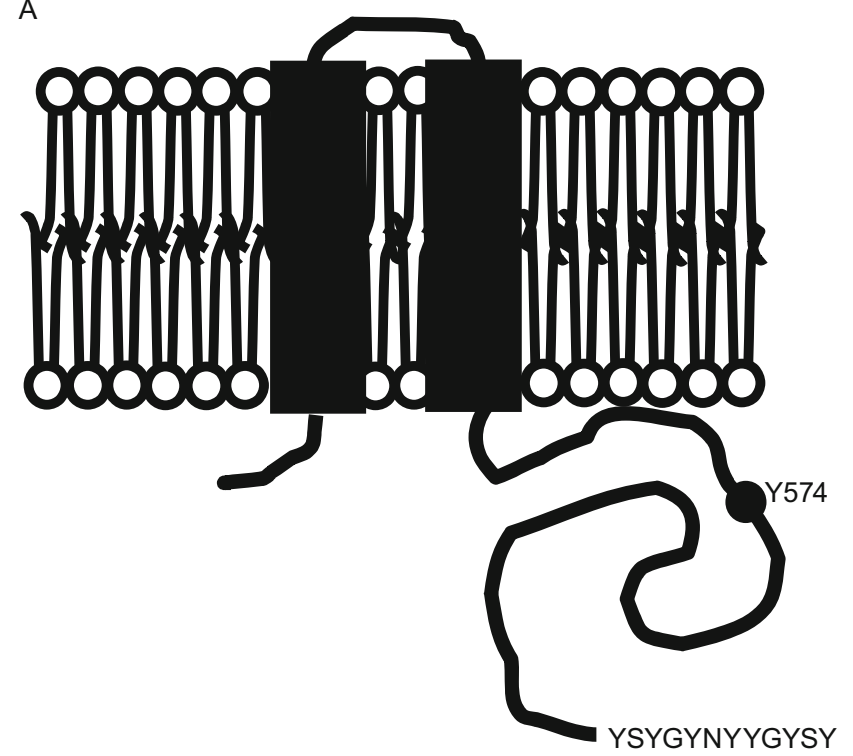

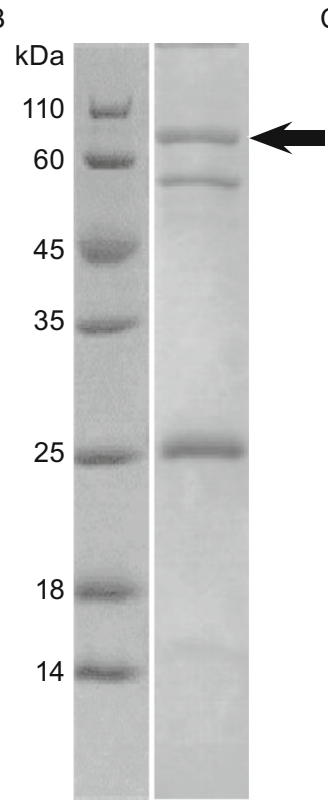

$\mathrm{C}$

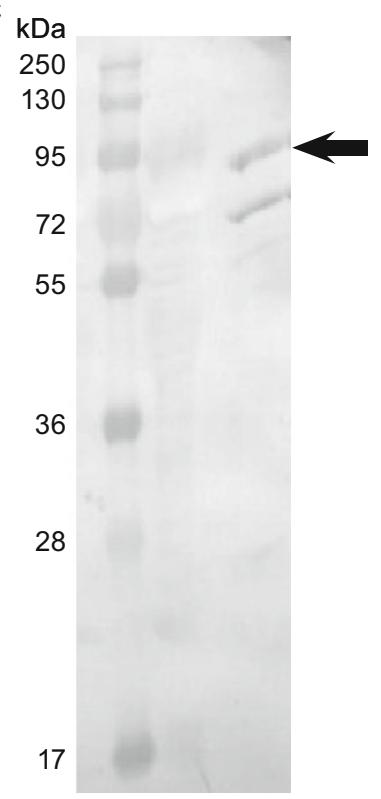

Figure 1. F2Y was incorporated at Tyr574 site of ETK-FL protein. (A) Topology diagram of an ETK protein in lipid bilayer, containing two transmembrane helices and a cytosolic tyrosine kinase domain including the auto-phosphorylation site $Y 574$ and tyrosine rich C-terminal tail; (B) Coomassie blue staining of purified ETK-Y574F $F_{2} Y$; (C) Western-blot of purified ETK-Y574F ${ }_{2} \mathrm{Y}$.

the $\mathrm{N}$-terminus was expressed in $E$. coli membrane through expression condition optimizations. Brief protein purification was conducted using Ni-NTA affinity chromatography. Coomassie blue staining (Fig. 1B) and Western blot (Fig. 1C) analysis of the SDS-PAGE on partially purified proteins demonstrated the expressed ETK- $Y 574 \mathrm{~F}_{2} \mathrm{Y}$ as $95 \mathrm{kDa}$ (black arrow), while the band around $58 \mathrm{kDa}$ was suspected as the truncated proteins stopping at amber stop codon introduced at the Tyr574 site, since the $\mathrm{His}_{6}$-tag was expressed at N-terminal of the ETK protein.

Retaining native membrane environment (without protein purification steps) is important for structure and function studies of the membrane proteins in their physiological condition. Here, the E. coli membrane fraction was obtained through ultracentrifugation method. Before the auto-phosphorylation analysis of ETK-FL with the presence of $\mathrm{Mg}^{2+}$ and ATP, the dephosphorylation treatment using PTP1B was implemented to remove any trace phosphorylations. Both the treatment by the PTP1B and $\mathrm{Mg}^{2+} /$ ATP required direct interactions between the small protease or compounds and the cytosolic domain of ETK-FL. However, multi-lamellar vesicles (MLV) will be formed from bacteria membrane spontaneously (Fig. 2A). The MLV would not only have high proportions of ETK-FL locate in inner vesicles, but also have dual orientations of the ETK-FL in outer vesicle, leaving only half populations of ETK-FL with the exposure of its cytosolic domain. Therefore, the liposome MLV provided huge physical barrier for accessibilities of soluble ligands or interaction partner proteins for membrane protein studies. To overcome this hindrance effect of MLV, the conventional procedures was to mix the compounds or protein partners with the target membrane protein before reaction, with extensive liposome re-distribution procedures, i.e. several cycles of sonicationfreeze-thaw. This procedure obviously requires complicate sample treatments, possibly having unexpected adverse influences to correct folding of proteins, but also makes it impossible to analyze kinetics of protein interactions. To improve accessibilities of soluble PTP1B or other compounds to ETK-FL in native lipid environment and also to simply the experimental procedure, poly-styrene-maleic-acid (SMA3000 3:1) compounds (purchased from Malvern Cosmeceutics company, UK and hydrated in authors' laboratory) were applied to wrap up the lipid bilayers to form uni-lamellar lipodisq (Fig. 2A).

To achieve suitable size of lipodisq, the appropriate concentrations of SMA3000 to E. coli native membrane need to be optimized. Briefly, the SMA3000 was added to the well dispersed bacteria membrane suspension, and then the sample was processed with several rounds of sonicationfreeze-thaw. Here, negative staining transmission electron microscopy (TEM) was applied to analyze the size of liposomes and vesicles. The $E$. coli native membrane was observed with diameter of about $300 \mathrm{~nm}$ (Fig. 2B, left). When the final concentration of $0.15 \mathrm{mmol} / \mathrm{L}$ poly-SMA3000 (3:1) was added into liposomes, the diameter of lipodisq was about $100 \mathrm{~nm}$ (Fig. 2B, middle). Average diameter of around 10-20 nm (Fig. 2B, right) can be reached, at final concentration of $0.45 \mathrm{mmol} / \mathrm{L}$ poly-SMA3000 (3:1). The observed 
A
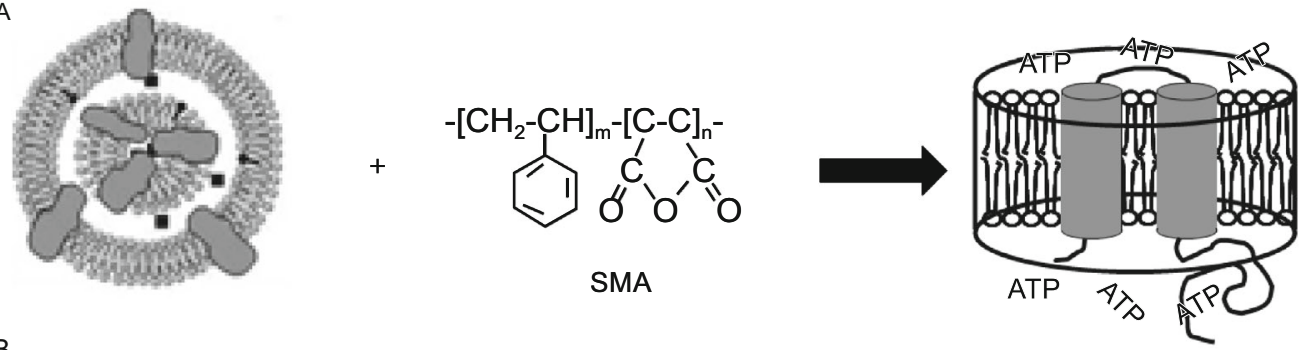

B

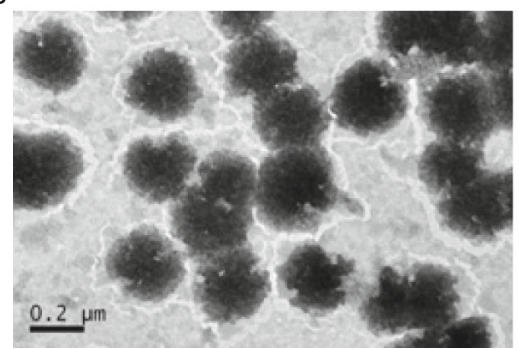

C

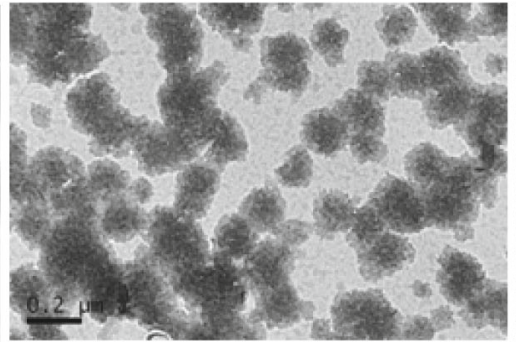

E

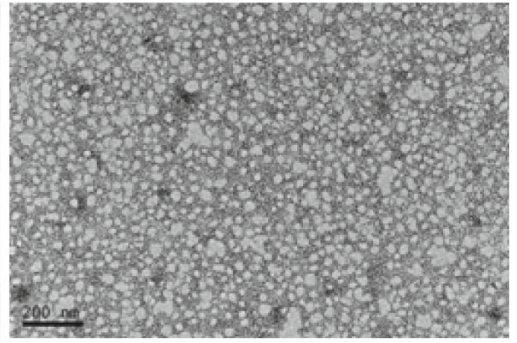

With SMA
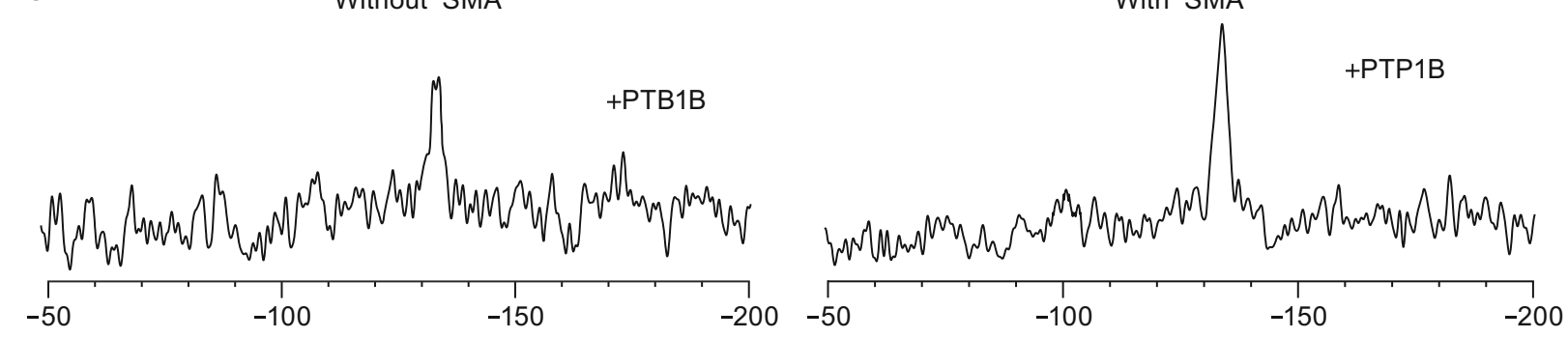

(100)

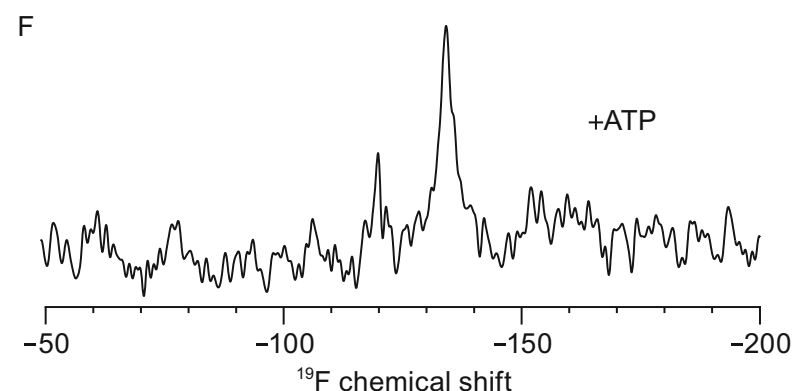

Figure 2. SMA wrapped uni-lamellar liposome elevated phosphorylation level of ETK-FL-Y574-F2Y. (A) Topology diagram of an ETK-FL in multi-lamellar lipid bilayer vesicles, composition of poly-SMA and topology diagram of an ETK protein in lipodisq; (B) Transmission electron microscopy pictures of native E. coli liposomes after freeze-thaw-bath sonication with addition of 0 , $10,30 \mu \mathrm{L}$ poly-SMA3000 (3:1). The $0.2 \mu \mathrm{m}$ bar was shown in the same size in the three pictures. (C) ${ }^{19} \mathrm{~F}$ NMR spectrum of ETK-

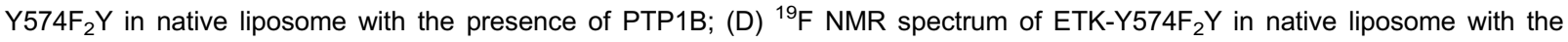
presence of ATP and $\mathrm{Mg}^{2+}$; (E) ${ }^{19} \mathrm{~F}$ NMR spectrum of ETK-Y574-F2Y in lipodisq with the presence of PTP1B; (F) ${ }^{19} \mathrm{~F} \mathrm{NMR}$ spectrum of ETK-Y574F2Y in lipodisq with the presence of ATP and $\mathrm{Mg}^{2+}$.

lipodisq with much smaller diameters were consistent with previously reported 10-20 nm size of SMA-lipid nanoparticles (Knowles et al., 2009).

Then, the ${ }^{19} \mathrm{~F}$ solid state NMR signals were acquired for phosphorylation analysis of ETK-Y574- $\mathrm{F}_{2} \mathrm{Y}$ to verify the improved substrate accessibilities of the membrane lipodisq. Using the previously described mixing procedures of E. coli membrane MLV and PTP1B, the trace phosphorylation were removed from the ETK-Y574- $\mathrm{F}_{2} \mathrm{Y}$ sample, and a single ${ }^{19} \mathrm{~F}$ NMR signal at $-133.0 \mathrm{ppm}$ was observed (Fig. $2 \mathrm{C}$ ), indicating the dephosphorylated $\mathrm{F}_{2} \mathrm{Y}$ (Li et al., 2013). Then, sodium vanadate $\left(\mathrm{Na}_{3} \mathrm{VO}_{4}\right)$ was added to the sample to suppress the PTP1B function. After addition of $\mathrm{Mg}^{2+}$ and ATP to ETK-Y574- $\mathrm{F}_{2} \mathrm{Y}$, initiating the auto-phosphorylation reaction, the bacteria cell membrane were pellet down using ultracentrifugation for further ${ }^{19} \mathrm{~F}$ solid state NMR analysis. As shown in Fig. $2 \mathrm{D}$, two ${ }^{19} \mathrm{~F}$ signals were observed, with one major peak at around $-133.2 \mathrm{ppm}$ 
(dephosphorylated state) and a weak peak around -119.4 ppm (phosphorylated state). This observation was consistent with previously observed small percentage of phosphorylated $\mathrm{Y} 574-\mathrm{F}_{2} \mathrm{Y}$ in soluble ETK-CD (Li et al., 2013). The observed tyrosine phosphorylation signals of ETK-FL protein in native bacteria membrane were similar as those of in vitro analysis of the purified ETK-CD. This verified that the ${ }^{19} \mathrm{~F}-\mathrm{F}_{2} \mathrm{Y}$ incorporation can be applied for further in situ tyrosine phosphorylation analysis of ETK or other membrane proteins.

With the prepared lipodisq of $E$. coli native membrane using SMA300 (3:1), the soluble phosphatase PTP1B or soluble substrate ATP, $\mathrm{Mg}^{2+}$ were added to the lipodisq samples directly. Then the samples were pelleted down using ultracentrifugation before solid state NMR analysis. Only one ${ }^{19} \mathrm{~F}$ signal of dephosphorylated state of ETK$\mathrm{Y} 574 \mathrm{~F}_{2} \mathrm{Y}$ was observed at $-133.8 \mathrm{ppm}$, in the presence of PTP1B (Fig. 2E). One major dephosphorylated signal and one minor phosphorylated signal were observed at -134.1 ppm or $-119.8 \mathrm{ppm}$ respectively with the presence of ATP and $\mathrm{Mg}^{2+}$ (Fig. 2F). Improved peak sensitivity of the phosphorylated signals $(-119.8 \mathrm{ppm})$ was observed for the lipodisq sample (Fig. 2F) than the liposome sample (Fig. 2D), probably due to the improved accessibility of ATP or $\mathrm{Mg}^{2+}$ in the SMA wrapped uni-lamellar liposome and consequent elevated phosphorylation for the ETK-FL- Y574- $F_{2} Y$. Therefore, size homogenous SMA wrap lipodisqs simplified the experiment procedure through improving the substrate accessing to kinase.

Here, we presented the advantage of correlational structure and function studies of membrane proteins in nano-size uni-lamellar lipodisq with SMA surrounding the lipid bilayer, even native cell membrane. Other alternative nano-size unilamellar membrane configurations, such as bicelles and nanodiscs, were reported to improve substrate accessibilities to two sides of an incorporated membrane protein in lipid bilayers (Raschle et al., 2010). However, specific lipid, detergents are required for bicelles formation, but resulting in a heterogeneous system (in size and composition) (Raschle et al., 2010). Apo-lipoprotein nanodiscs, composing of a lipid core surrounded by an amphipathic membrane scaffold protein (MSP) (Bayburt and Sligar, 2003, 2010), had the advantage that they could be made from a variety of lipid mixtures, and was relatively mono-dispersed in size upon protein incorporation. Nevertheless, nanodiscs still required detergents for protein incorporation, and could not be directly applied for native membrane system. Moreover, presence of the MSP might bring unpredictable interferences to incorporated proteins or specific interactions to lipids in core. Besides the well integrity maintenance of the native environment and the integral membrane proteins in uni-lamellar lipodisq, the improved accessibility of soluble partner to SMA based lipodisqs in homogenous size provided much convenience for further receptor ligand binding, enzymology analysis and protein-protein interaction analysis in native cell membrane environment using ${ }^{19} \mathrm{~F}$ solid state NMR or other biophysical methods. Therefore, size homogenous SMA based lipodisqs can not only improve accessibility of soluble partner, but also maintain integrity of the native environment for the integral membrane proteins.

\section{FOOTNOTES}

Authors are grateful for plasmid pEvol-F2Y provided by Dr. Jiangyun Wang, compounds F2Y provided by Dr. Fan Yang, in Institute of Biophsycis, Chinese Academy of Sciences, Beijing, China. This work was supported by the National Basic Research Program (973 Program) (Nos. 2013CB910200 and 2015CB910100), and the National Natural Science Foundation of China (Grant Nos. 31100563, 31300685 and U1332210, and Strategic Priority Research Program of CAS: XDB08030302).

Dong Li, Juan Li, Yonglong Zhuang, Longhua Zhang, Ying Xiong, Pan Shi and Changlin Tian declare that they have no conflict of interest. This article does not contain any studies with human or animal subjects performed by the any of the authors.

Dong $\mathrm{Li}^{1}$, Juan $\mathrm{Li}^{1}$, Yonglong Zhuang ${ }^{3}$, Longhua Zhang ${ }^{1}$, Ying Xiong ${ }^{1}$, Pan Shi ${ }^{2 \bowtie}$, Changlin Tian ${ }^{1,2 \bowtie}$

${ }^{1}$ Hefei National Laboratory for Physical Science at the Microscale School of Life Science, University of Science and Technology of China, Hefei 230026, China

2 High Magnetic Field Laboratory, Chinese Academy of Sciences, Hefei 230031, China

${ }^{3}$ Department of Chemistry, Anhui University, Hefei 230027, China

$\bowtie$ Correspondence: shipan@hmfl.ac.cn (P. Shi), cltian@ustc.edu. cn (C. Tian)

\section{OPEN ACCESS}

This article is distributed under the terms of the Creative Commons Attribution License which permits any use, distribution, and reproduction in any medium, provided the original author(s) and the source are credited.

\section{REFERENCES}

Barnes CO, Pielak GJ (2011) In-cell protein NMR and protein leakage. Proteins 79:347-351

Bayburt TH, Sligar SG (2003) Self-assembly of single integral membrane proteins into soluble nanoscale phospholipid bilayers. Protein Sci 12:2476-2481

Bayburt TH, Sligar SG (2010) Membrane protein assembly into Nanodiscs. FEBS Lett 584:1721-1727

Hammill JT, Miyake-Stoner S, Hazen JL, Jackson JC, Mehl RA (2007) Preparation of site-specifically labeled fluorinated proteins for 19F-NMR structural characterization. Nat Protoc 2:2601-2607 Hunter T (2009) Tyrosine phosphorylation: thirty years and counting. Curr Opin Cell Biol 21:140-146

Jackson JC, Hammill JT, Mehl RA (2007) Site-specific incorporation of a (19) F-amino acid into proteins as an NMR probe for characterizing protein structure and reactivity. J Am Chem Soc 129:1160-1166

Johnson H, White FM (2012) Toward quantitative phosphotyrosine profiling in vivo. Semin Cell Dev Biol 23:854-862 
Knowles TJ, Finka R, Smith C, Lin YP, Dafforn T, Overduin M (2009) Membrane proteins solubilized intact in lipid containing nanoparticles bounded by styrene maleic acid copolymer. J Am Chem Soc 131:7484-7485

Lee DC, Zheng J, She YM, Jia Z (2008) Structure of Escherichia coli tyrosine kinase Etk reveals a novel activation mechanism. EMBO J 27:1758-1766

Li F, Shi P, Li J, Yang F, Wang T, Zhang W, Gao F, Ding W, Li D, Li J et al (2013) A genetically encoded 19F NMR probe for tyrosine phosphorylation. Angew Chem Int Ed Engl 52:3958-3962

Orwick MC, Judge PJ, Procek J, Lindholm L, Graziadei A, Engel A, Grobner G, Watts A (2012) Detergent-free formation and physicochemical characterization of nanosized lipid-polymer complexes: Lipodisq. Angew Chem Int Ed Engl 51:4653-4657

Orwick-Rydmark M, Lovett JE, Graziadei A, Lindholm L, Hicks MR, Watts A (2012) Detergent-Free incorporation of a seven-transmembrane receptor protein into nanosized bilayer Lipodisq particles for functional and biophysical studies. Nano lett 12:4687-4692

Raschle T, Hiller S, Etzkorn M, Wagner G (2010) Nonmicellar systems for solution NMR spectroscopy of membrane proteins. Curr Opin Struct Biol 20:471-479

Shi P, Xi Z, Wang H, Shi C, Xiong Y, Tian C (2010) Site-specific protein backbone and side-chain NMR chemical shift and relaxation analysis of human vinexin $\mathrm{SH} 3$ domain using a genetically encoded 15N/19F-labeled unnatural amino acid. Biochem Biophys Res Commun 402:461-466

Shi P, Wang H, Xi Z, Shi C, Xiong Y, Tian C (2011) Site-specific (1)(9) F NMR chemical shift and side chain relaxation analysis of a membrane protein labeled with an unnatural amino acid. Protein Sci 20:224-228

Shi P, Li D, Chen H, Xiong Y, Wang Y, Tian C (2012) In situ 19F NMR studies of an E. coli membrane protein. Protein Sci 21:596-600

Electronic supplementary material The online version of this article (doi:10.1007/s13238-014-0129-x) contains supplementary material, which is available to authorized users. 